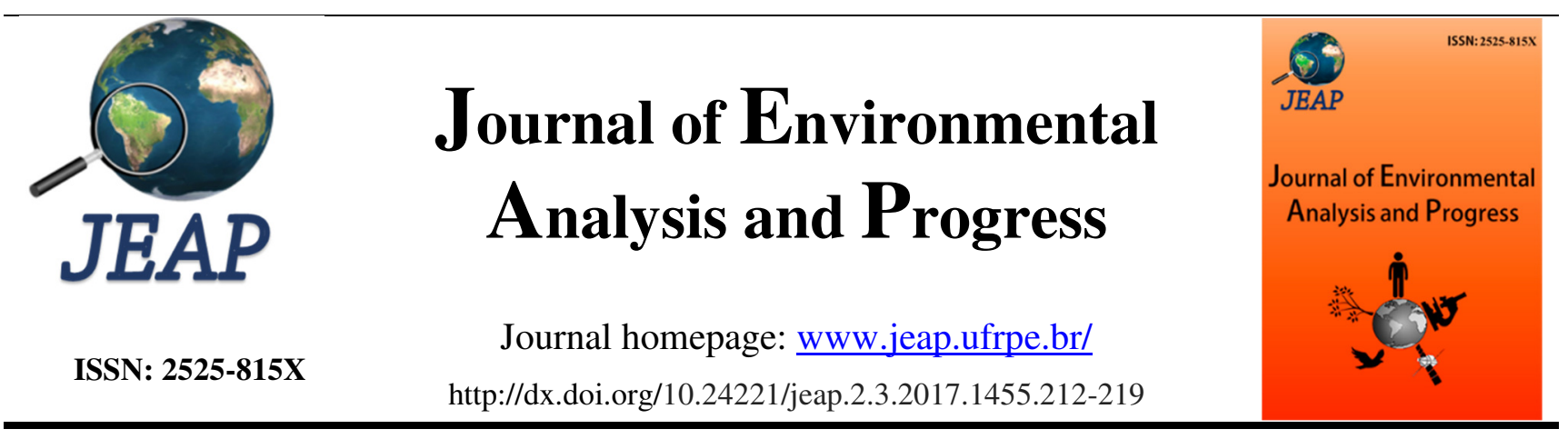

\title{
Emergência e crescimento inicial de Amburana cearensis sob aumento da temperatura e dióxido de carbono
}

\section{Emergency and initial growth of Amburana cearensis under increase temperature and carbon dioxide}

\author{
Danielle Carolina Campos da Costa ${ }^{\mathrm{a}}$, Jaciara de Souza Bispo ${ }^{\mathrm{b}}$, Gilmara Moreira de Oliveira ${ }^{\mathrm{c}}$, \\ Francislene Angelotti ${ }^{\mathrm{d}}$, Bárbara França Dantas ${ }^{\mathrm{e}}$ \\ a Embrapa Semiárido. Rodovia BR-428, Km 152, s/n, Zona Rural, Petrolina, Pernambuco, Brasil. CEP: 56302-970. E- \\ mail: daniellecarolina_58@hotmail.com; jaciarabispo@ymail.com; gilmara_5@hotmail.com; \\ francislene.angelotti@embrapa.br; bfdantas@embrapa.br.
}

\section{A R T I C L E I N F O}

Recebido 29 Jun 2017

Aceito 11 Jul 2017

Publicado 31 Jul 2017

\begin{abstract}
A B S T R A C T
The concentration of carbon dioxide $\left[\mathrm{CO}_{2}\right]$ in the atmosphere has increased in recent years, as a consequence may have direct and indirect effects on agroecosystems, and one of the biomes that will suffer most from this change will be the Caatinga. Amburana cearensis is a species native to this biome and widely used for medicinal, wood, ornamental and forage purposes. The objective of this study was to evaluate the effect of the increase of $\left[\mathrm{CO}_{2}\right]$ on the emergence and initial growth of $A$. cearensis seedlings. The experiment was conducted in Fitotron type growth chambers with temperature control, $\left[\mathrm{CO}_{2}\right]$, humidity and photoperiod. Seeds were seeded in tubes containing a commercial substrate and submitted to two temperature regimes $\left(18-24-30^{\circ} \mathrm{C}\right.$ and $\left.22-28-34^{\circ} \mathrm{C}\right)$ and two $\left[\mathrm{CO}_{2}\right]$ (390 and 770 $\mathrm{ppm}$ ) for 56 days. The percentage of emergency, average emergence time, mean emergency speed, emergency speed index, leaf number, lap diameter, relative chlorophyll index, length, fresh weight and total dry matter of the seedlings were evaluated. Evaluating in an isolated way, the temperature and $\mathrm{CO}_{2}$ concentration showed an influence on the total fresh weight of the A. cearensis seedlings. The interaction between temperature and $\left[\mathrm{CO}_{2}\right]$ did not affect the emergence of seedlings. In initial seedling growth, only the variables diameter of the colon and dry weight were influenced by this interaction
\end{abstract}

Keywords: Caatinga, climate change, umburana-de-cheiro.

R E S U M O
A concentração de dióxido de carbono $\left[\mathrm{CO}_{2}\right]$ na atmosfera tem aumentado nos
últimos anos, como consequência poderá causar efeitos diretos e indiretos nos
agroecossistemas, e um dos biomas que mais sofrerá com esta mudança será a
Caatinga. A Amburana cearensis é uma espécie nativa desse bioma e muito
utilizada para fins medicinais, madeireiro, ornamental e forrageiro. Este estudo
objetivou avaliar o efeito do aumento da $\left[\mathrm{CO}_{2}\right]$ sobre a emergência e crescimento
inicial de plântulas de A. cearensis. O experimento foi conduzido em câmaras de
crescimento, tipo Fitotron, com controle de temperatura, [CO $\left.\mathrm{CO}_{2}\right]$, umidade e
fotoperíodo. As sementes foram semeadas em tubetes contendo substrato comercial
e submetidas a dois regimes de temperatura $\left(18-24-30^{\circ} \mathrm{C}\right.$ e $\left.22-28-34^{\circ} \mathrm{C}\right)$ e duas
[CO $\left.\mathrm{CO}_{2}\right]$ ( 390 e 770 ppm), durante 56 dias. Foram avaliados porcentagem de
emergência, tempo médio de emergência, velocidade média de emergência, índice
de velocidade de emergência, número de folhas, diâmetro do colo, índice relativo
de clorofila, comprimento, peso da massa fresca e seca total das plântulas. Quando


avaliados de maneira isolada, a temperatura e a concentração de $\mathrm{CO}_{2}$ apresentaram influência sobre o peso da massa fresca total das plântulas de A. cearensis. A interação entre temperatura e $\left[\mathrm{CO}_{2}\right]$ não influenciou na emergência de plântulas. No crescimento inicial de plântulas, apenas as variáveis diâmetro do colo e peso da massa seca foram influenciadas por esta interação.

Palavras-Chave: Caatinga, mudanças climáticas, umburana-de-cheiro.

\section{Introdução}

As projeções climáticas indicam que o aumento da emissão dos gases de efeito estufa, provocado por ações antropogênicas, é um dos fatores responsáveis pela elevação da temperatura terrestre. Os estudos têm indicado que nos últimos 250 anos, a concentração de dióxido de carbono $\left[\mathrm{CO}_{2}\right]$ na atmosfera aumentou de 290 para 379 partes por milhão (ppm), com a previsão de atingir níveis acima de 500 ppm em 2100. Em consequência deste aumento, tem sido observado um aumento na temperatura média do ar. $\mathrm{E}$ os cenários climáticos futuros indicam que poderá ocorrer um aumento entre $1,8^{\circ} \mathrm{C}$ a $6,4^{\circ} \mathrm{C}$ até o final deste século (IPCC, 2014). Para o Semiárido brasileiro a projeção de aumento será de $3,5^{\circ} \mathrm{C}$ a $4,5^{\circ} \mathrm{C}$, com redução na precipitação, sendo considerada uma das regiões brasileiras mais vulneráveis às mudanças climáticas globais (Ambrizzi \& Araújo, 2012).

As mudanças climáticas poderão provocar modificações nos ecossistemas terrestres promovendo alterações na vegetação. A temperatura, o $\mathrm{CO}_{2}$ e outros fatores ambientais afetam todas as fases de desenvolvimento das plantas, desde a germinação das sementes até o crescimento e estabelecimento das plantas, causando interferência direta nessas etapas (Brown, Valone \& Curtin, 1997; Walther et al., 2002; Buckeridge, 2008). Pesquisas afirmam que o $\mathrm{CO}_{2}$ interfere no metabolismo e na germinação das sementes, reduzindo a taxa de respiração das mesmas (Aguiar et al., 2012). Além disso, a temperatura é um fator de grande relevância no processo de germinação das sementes, uma vez que atua na velocidade de absorção de água e nas reações bioquímicas afetando consequentemente a velocidade e a uniformidade de germinação (Martins et al., 2008; Passos et al., 2008).

Para a Caatinga, um dos biomas mais vulneráveis ao aquecimento global (IPCC, 2014), o aumento da temperatura poderá causar alteração na distribuição da vegetação e acelerar o processo de desertificação (Ambrizzi et al., 2013; Salazar et al., 2007). O aumento da temperatura e redução no índice pluviométrico poderão causar impactos nas características e distribuição da vegetação com intensificação da aridez da região até final deste século (Marengo, 2006; Salazar et al., 2007), causando sérios problemas ao uso da terra (Magalhães, 2009; 2013) e acelerando o processo de desertificação (Angelotti et al., 2009). De maneira geral, o aumento da temperatura e da concentração de $\mathrm{CO}_{2}$, pode favorecer $\mathrm{o}$ encurtamento no ciclo do crescimento e a elevação da respiração do tecido vegetal (Taiz \& Zeiger, 2009; Streck, 2005).

Neste bioma são encontradas espécies vegetais de grande relevância econômica com diversidade de usos, sendo utilizadas para a forragem, madeira, energia, ornamentação e produtos fitoterápicos (Cunha et al., 2009).

Amburana cearensis (Allemão) A.C. Smith pertence à família Fabaceae, é uma espécie nativa da Caatinga, conhecida como amburana ou umburana (Maia, 2006; Lorenzi \& Matos, 2008). Tem ocorrência natural no Nordeste do Brasil, nos Estados do Espírito Santo e Minas Gerais, na Floresta Pluvial do Vale do Rio Doce e nos afloramentos calcários e matas decíduas dos Estados de Mato Grosso, Goiás, Tocantins, Mato Grosso do Sul e São Paulo (Lorenzi, 2002). A árvore de umburana é recomendada para estudos que busquem a recuperação de áreas degradadas (Maia, 2004).

As mudanças climáticas poderão afetar também a dinâmica populacional de espécies influenciando a germinação de sementes através da maturação, da massa ou até mesmo a persistência dessas sementes no solo (Walck et al., 2011).

Levando em consideração o grande valor econômico, forrageiro, madeireiro, medicinal da espécie em estudo para população são necessários estudos sobre a emergência, crescimento inicial de plântulas e formação de mudas destas espécies frente às mudanças climáticas. Assim, objetivouse com este estudo avaliar o efeito do aumento da temperatura e concentração de $\mathrm{CO}_{2}$ sobre a emergência e o crescimento inicial de plântulas de A. cearensis.

\section{Material e Métodos}

As sementes de A. cearensis foram coletadas na comunidade de Jutaí, no interior de Lagoa Grande-PE, com as seguintes coordenadas W 08 36 ' 55,20" S 4007' 13,6”.

O experimento foi conduzido na Embrapa Semiárido, Petrolina-PE, em câmaras de crescimento, tipo Fitotron, com controle de temperatura, $\left[\mathrm{CO}_{2}\right]$, luz e umidade. $\mathrm{O}$ delineamento experimental utilizado foi o inteiramente casualizado, com os tratamentos distribuídos em arranjo fatorial $2 \times 2$ (2 regimes 
de temperaturas $\mathrm{x} 2$ concentrações de $\mathrm{CO}_{2}$ ) com quatro repetições de 24 sementes (tubetes), com rega diária até obtenção da capacidade de campo.

Os regimes de temperatura consistiram em $18-24-30^{\circ} \mathrm{C}\left(18^{\circ} \mathrm{C}\right.$ : das $18 \mathrm{~h}$ às $6 \mathrm{~h} ; 24^{\circ} \mathrm{C}$ : das $6 \mathrm{~h}$ às $12 \mathrm{~h} ; 30^{\circ} \mathrm{C}$ : das $12 \mathrm{~h}$ às $16 \mathrm{~h} ; 24^{\circ} \mathrm{C}$ : das $16 \mathrm{~h}$ às $18 \mathrm{~h})$ e $22-28-34^{\circ} \mathrm{C}\left(22^{\circ} \mathrm{C}\right.$ : das $18 \mathrm{~h}$ às $6 \mathrm{~h} ; 28^{\circ} \mathrm{C}$ : das $6 \mathrm{~h}$ às $12 \mathrm{~h} ; 34^{\circ} \mathrm{C}$ : das $12 \mathrm{~h}$ às $16 \mathrm{~h} ; 28^{\circ} \mathrm{C}$ : das $16 \mathrm{~h}$ às $18 \mathrm{~h}$ ), baseado nas temperaturas médias da região. Estes regimes térmicos foram combinados com duas concentrações de $\mathrm{CO}_{2}: 390 \mathrm{ppm}$, considerada a concentração atual e 770 ppm, concentração futura para 2100 no cenário RCP8.5 (cenário mais pessimista) de acordo com previsões do IPCC (2014). As condições ambientais nas câmaras de crescimento foram monitoradas automaticamente, o $\mathrm{CO}_{2}$ foi injetado por cilindro com $99,8 \%$ de $\mathrm{CO}_{2}$, pressurizado a 58,3 kg.F.cm ${ }^{-2}$.

Foram analisadas as seguintes variáveis:

Porcentagem de emergência (E\%)- $\mathrm{f}$ oi calculada a partir de contagens diárias de plântulas emergidas, de quatro repetições com 24 sementes durante 28 dias (Equação 1). Foram consideradas plântulas emergidas as que apresentaram emissão de hipocótilo visível acima do substrato. A partir dos dados obtidos nas contagens diárias foram calculados:

Tempo médio de emergência (TME) - média do tempo, em dias, para que as sementes germinem. Trata-se de uma média calculada para distribuições de frequência, em que o número de sementes germinadas representa a frequência de germinação. $\mathrm{O}$ cálculo é uma média ponderada, sendo o número de sementes germinadas o peso de ponderação do tempo. (Equação 2) (Labouriau, 1983; Santana \& Ranal, 2000).

Velocidade média de emergência (VME) calculada através da contagem diária, considerada como inverso do tempo médio de germinação (Equação 3) (Kotowski, 1926; Santana \& Ranal, 2000).

Índice de velocidade de emergência (IVE) calculado a partir da soma de sementes germinadas dividido pelo tempo necessário para que isso acontecesse (Equação 4) (Maguire, 1962; Santana \& Ranal, 2000).

$$
\begin{aligned}
& G=\frac{\sum_{i=1}^{k} n i}{A} * 100 \\
& T M G=\frac{\sum_{i=1}^{k} n i . t i}{\sum_{i=1}^{k} n i} \\
& V M G=\frac{\sum_{i}^{k} n i}{\sum_{i=1}^{k} n i . t i}
\end{aligned}
$$

Equação 2

Equação 3

$$
I V G=\sum_{i=1}^{k} \frac{N i}{t i}
$$

Equação 4

onde $\mathrm{K}$ = último dia de observação; $\mathrm{A}$ = Número total de sementes colocadas para germinar; $\mathrm{ni}=$ número não acumulado de sementes germinadas; ti $=$ número de dias; $\mathrm{Ni}=$ número acumulado de sementes germinadas.

\section{Crescimento inicial de plântulas}

As plântulas foram mantidas nas câmaras de crescimento até os 56 dias após a semeadura, sendo retiradas quatro plântulas por repetição para a realização das seguintes avaliações:

Número de folhas - contagem do número de folhas sendo consideradas apenas as folhas expandidas;

Diâmetro do colo (mm) - avaliou-se o diâmetro do colo das plântulas usando paquímetro digital, os resultados foram expressos em milímetros;

Índice relativo de clorofila (IRC) - Para a avaliação do teor de clorofila em folhas foi usado clorofilômetro (ClorofiLOG 1030), sendo avaliada uma folha por plântula.

Comprimento total de plântulas $(\mathrm{cm})$ - foi medido o comprimento da parte aérea e raiz, com o auxílio de régua milimetrada, os resultados foram expressos em centímetros.

Fitomassa de plântulas (g) - As plântulas foram pesadas em balança analítica de precisão $0,001 \mathrm{~g}$, com valores expressos em gramas. Posteriormente foram submetidas a secagem em estufa com circulação de ar forçada a $65^{\circ} \mathrm{C}$ até massa constante, sendo novamente pesadas para a obtenção da massa seca.

Análise estatística - Os dados obtidos de \%E foram transformados em arco seno da raiz quadrada de x/100 (Santana \& Ranal, 2000). Os dados foram submetidos à análise de variância (ANOVA) e as médias comparadas pelo teste de Scott-Knott a 5\% de probabilidade (Ferreira, 2008).

\section{Resultados}

Houve interação entre os fatores (Temperatura $\mathrm{x} \mathrm{CO}_{2}$ ) para as variáveis: diâmetro do colo (DC) e peso da massa seca total (PST). A interação temperatura e a concentração de $\mathrm{CO}_{2}$ não foi significativa para a emergência de plântulas, tempo médio de emergência, velocidade média de emergência e índice de velocidade de emergência. Porém, quando analisados separadamente, estes fatores foram significativos, para o peso da massa fresca total (PFT) (Tabela $1)$. 
Tabela 1. Resumo da análise de variância, pelo quadrado médio, referentes aos valores de Porcentagem de emergência (E), Tempo médio de emergência (TME), Velocidade média de emergência (VME), Índice de velocidade de emergência (IVE), número de folhas (NF), diâmetro do colo (DC), Índice relativo de clorofila (IRC), comprimento total (CT), peso da massa fresca total (PFT) e peso da massa seca total (PST) de plântulas de Amburana cearensis mantidas em ambientes com diferentes regimes de temperatura $\left(18-24-30^{\circ} \mathrm{C}\right.$ e $22-28$ $34^{\circ} \mathrm{C}$ ), concentrações de $\mathrm{CO}_{2}$ (390 ppm e 770 ppm), Petrolina-PE, em 2016.

\section{Quadrado médio}

\begin{tabular}{|c|c|c|c|c|}
\hline Fonte de variação & Temperatura (T) & $\mathrm{CO}_{2}$ & $\mathrm{~T} \times \mathrm{CO}_{2}$ & CV $(\%)$ \\
\hline E & $113,09^{\text {ns }}$ & $66,11^{\mathrm{ns}}$ & $18,45^{\mathrm{ns}}$ & 15,36 \\
\hline TME & $4,39^{\mathrm{ns}}$ & $6,10^{\mathrm{ns}}$ & $1,62^{\mathrm{ns}}$ & 7,19 \\
\hline VME & $0,00006^{\mathrm{ns}}$ & $0,00008^{\mathrm{ns}}$ & $0,00001^{\mathrm{ns}}$ & 8,00 \\
\hline IVE & $0,03^{\text {ns }}$ & $0,09^{\text {ns }}$ & $0,03^{\text {ns }}$ & 23,22 \\
\hline NF & $0,14^{\mathrm{ns}}$ & $0,01^{\mathrm{ns}}$ & $0,39^{\text {ns }}$ & 9,42 \\
\hline DC & $0,19^{\text {ns }}$ & $1,16^{* *}$ & $0,32^{*}$ & 8,66 \\
\hline IRC & $2,38^{\mathrm{ns}}$ & $27,75^{\mathrm{ns}}$ & $0,59^{\text {ns }}$ & 12,23 \\
\hline $\mathrm{CT}$ & $0,56^{\mathrm{ns}}$ & $3,42^{\text {ns }}$ & $2,52^{\mathrm{ns}}$ & 4,78 \\
\hline PFT & $22,08^{* *}$ & $20,76^{* *}$ & $1,84 \mathrm{~ns}$ & 15,68 \\
\hline PST & $1,44^{* *}$ & $0,08^{\mathrm{ns}}$ & $2,15^{* *}$ & 14,54 \\
\hline
\end{tabular}

$\mathrm{CV}=$ coeficiente de variação; ns = não significativo, ${ }^{* *}$ significativo ao nível de $1 \%$ de probabilidade, $*$ significativo ao nível de $5 \%$ de probabilidade pelo teste de Scott-Knott.

O menor diâmetro do colo foi observado em plântulas de A. cearensis mantidas em ambiente com temperatura de $18-24-30^{\circ} \mathrm{C}$ e concentração de 390 ppm de $\mathrm{CO}_{2}$. As plântulas, mantidas na temperatura de $22-28-34^{\circ} \mathrm{C}$, não apresentaram influência do aumento da concentração de $\mathrm{CO}_{2}$ (Tabela 2).

Tabela 2. Diâmetro do colo de plântulas e peso da massa seca total de plântulas de Amburana cearensis mantidas em ambientes com diferentes regimes de temperatura $\left(18-24-30^{\circ} \mathrm{C}\right.$ e $\left.22-28-34^{\circ} \mathrm{C}\right)$, concentrações de $\mathrm{CO}_{2}$ (390 ppm e 770 ppm). Petrolina-PE, em 2017.

\begin{tabular}{|c|c|c|}
\hline \multicolumn{3}{|c|}{ Diâmetro do colo (mm) } \\
\hline Temperatura/ $\left[\mathrm{CO}_{2}\right]$ & 390 ppm & 770 ppm \\
\hline $18-24-30^{\circ} \mathrm{C}$ & $2,30 \mathrm{bB}$ & $3,12 \mathrm{aA}$ \\
\hline $22-28-34^{\circ} \mathrm{C}$ & $2,80 \mathrm{aA}$ & $3,05 \mathrm{aA}$ \\
\hline \multicolumn{3}{|c|}{ Peso da massa seca total (g) } \\
\hline Temperatura/ $\left[\mathrm{CO}_{2}\right]$ & 390 ppm & 770 ppm \\
\hline $18-24-30^{\circ} \mathrm{C}$ & $2,31 \mathrm{aB}$ & $2,90 \mathrm{aA}$ \\
\hline $22-28-34^{\circ} \mathrm{C}$ & $2,44 \mathrm{aA}$ & $1,56 \mathrm{bB}$ \\
\hline
\end{tabular}

Médias seguidas pela mesma letra minúscula na coluna e maiúscula na linha não diferem entre si pelo teste de Scott-Knott a 5\% de probabilidade.

Para o peso da massa seca total foi observado que as plântulas em ambiente $\mathrm{o}$ aumento da temperatura e da concentração de $\mathrm{CO}_{2}$ proporcionaram menor peso. Entretanto, sob uma temperatura de $18-24-30^{\circ} \mathrm{C}$, o aumento da $\left[\mathrm{CO}_{2}\right]$ ocasionou um aumento no peso da massa seca total (Tabela 2).
Quando avaliados de maneira isolada, a temperatura e concentração de $\mathrm{CO}_{2}$ apresentaram influência sobre o peso da massa fresca total das plântulas de A. cearensis. A elevação da temperatura reduziu o peso da massa fresca das plântulas. No entanto, o ambiente com maior concentração de $\mathrm{CO}_{2}$ proporcionou aumento do peso da massa fresca (Figura 1). 

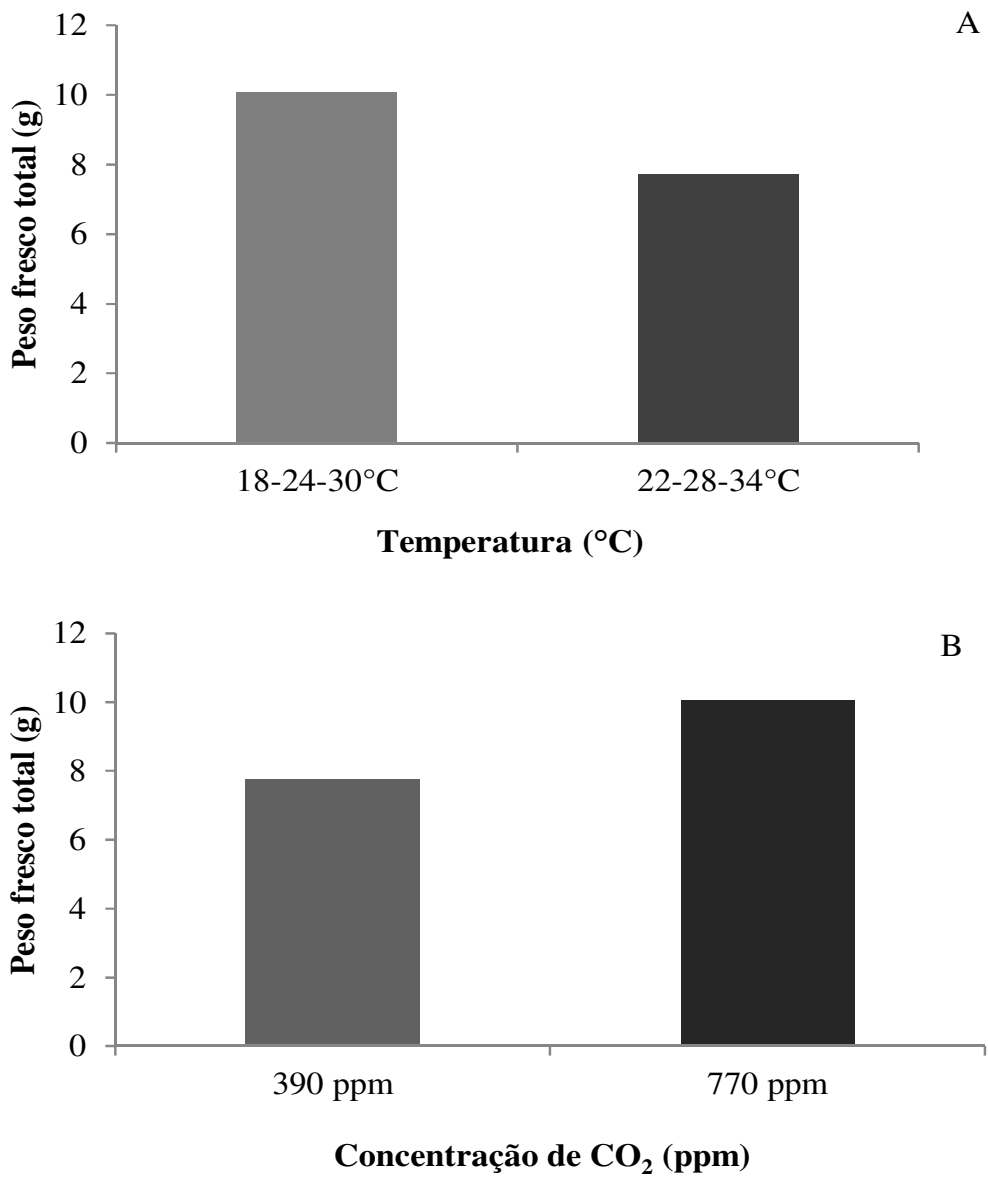

Figura 1. Peso de massa fresca de plântulas de Amburana cearensis mantidas em ambientes com diferentes regimes de temperatura $\left(18-24-30^{\circ} \mathrm{C}\right.$ e $\left.22-28-34^{\circ} \mathrm{C}\right)(\mathrm{A})$ e concentrações de $\mathrm{CO}_{2}$ (390 ppm e $\left.770 \mathrm{ppm}\right)(\mathrm{B})$.

\section{Discussão}

A germinação é um processo que necessita de consumo de energia. Nas células vivas, os principais processos para se obter energia são a respiração e a fermentação. Estes dois processos envolvem trocas de gases $\left(\mathrm{O}_{2}\right.$ e $\left.\mathrm{CO}_{2}\right)$ entre as células e o meio. Logo, a germinação é intensamente atingida pela atmosfera que cerca a semente (Castro, Kluge \& Peres, 2005), sendo que as altas temperaturas promovem um desbalanço na entrada e saída de $\mathrm{CO}_{2}$ pelas plantas (Atkin, Scheurwater \& Pons, 2007). Neste estudo não foi verificado o efeito do aumento da temperatura e da $\left[\mathrm{CO}_{2}\right]$ na germinação das sementes.

Além disso, a fisiologia das plantas sofre influência direta da concentração de $\mathrm{CO}_{2}$ atmosférico, sendo esta a fonte primária de carbono para fotossíntese, tornando-se um fator limitante neste processo (Martinez et al., 2015). Em geral, o efeito da concentração de $\mathrm{CO}_{2}$ sobre o crescimento das plantas é dependente da temperatura (Reyenga et al., 1999). Com o aumento da temperatura de crescimento, a resposta ao elevado nível de $\mathrm{CO}_{2}$ também aumenta, sendo que a temperatura ótima para o crescimento e desenvolvimento é bastante variável entre as espécies (Martinez et al., 2015).

As temperaturas acima da faixa ótima prejudicam o crescimento com consequente diminuição da taxa de fotossíntese e da concentração da enzima Rubisco e da clorofila (Yamasaki et al., 2002). Além disso, estudos apontam que o $\mathrm{CO}_{2}$ tem grande importância para a fotossíntese e possibilita a influência nos processos metabólicos das plantas (Rezende et al., 2015) e que um dos principais efeitos de deste gás no crescimento das plantas está numa aceleração do crescimento inicial das mesmas (Hattenschwiler et al., 1997; Würth \& Winter; Körner, 1998; Centritto, Lee \& Jarvis, 1999).

De acordo com Crafs-Brandner \& Salvucci (2000), a fotossíntese e o crescimento de plantas podem ter prejuízos causados pela elevação da temperatura, uma vez que a enzima Rubiscoativase tem a função de regular a atividade da Rubisco e é muito sensível ao aumento da temperatura tornando-se inativa com o estresse térmico.

No entanto, pesquisas indicam que aumento da temperatura está associado à aceleração do crescimento, podendo reduzir o ganho de 
biomassa (Lobell \& Gourdji, 2012). Assim, como a semente está permanentemente exposta à temperatura, este fator pode se constituir em um forte indicador do ambiente e de sua variação a longo e curto prazo (Cardoso, 2011).

Por outro lado, o ambiente enriquecido com $\mathrm{CO}_{2}$ tende a elevar a biomassa das plantas. Entretanto, para algumas espécies, se o aumento da concentração de $\mathrm{CO}_{2}$ for acompanhado de aumento da temperatura do ar, poderá ocorrer um aumento da respiração (Taiz \& Zeiger, 2013). Para as plântulas de A. cearensis, o peso da massa fresca aumentou em ambiente enriquecido com $\mathrm{CO}_{2}$.

O metabolismo das plantas tende a ser alterado em níveis elevados de $\mathrm{CO}_{2}$, de modo que, mais assimilados são desviados para as raízes havendo aumento da respiração radicular, ocasionando perda de produtos fotossintéticos, afetando o crescimento, resistência aos fatores de estresse e também a síntese de compostos de defesa (Larcher, 2006; Sage, 2002).

Portanto, a elevação da concentração de $\mathrm{CO}_{2}$ pode promover mudanças fisiológicas nas plantas e indiretamente causar modificações no funcionamento, estrutura e constituição das comunidades vegetais (Zavaleta et al., 2003; Zavaleta, 2006). Além disso, a viabilidade das sementes pode ser comprometida pela elevação da temperatura do ar e consequentemente elevação da temperatura no solo (Ooi, Auld \& Denham, 2009). Isto porque, a germinação e o estabelecimento de plântulas requerem uma de série técnicas e conhecimentos que estejam relacionados à produção, dispersão, características morfofisiológicas das sementes, tais como tolerância a uma ampla faixa de condições ambientais extremas (Meiado, 2012).

Os resultados obtidos a partir destes estudos de emergência e crescimento de plântulas de $A$. cearensis poderão contribuir de maneira significativa na prática de proteção e preservação dessa espécie.

\section{Conclusão}

A emergência de sementes de Amburana cearensis não foi influenciada pela interação temperatura $\mathrm{x}\left[\mathrm{CO}_{2}\right]$. No crescimento inicial de plântulas apenas as variáveis diâmetro do colo e peso da massa seca foram influenciadas por esta interação.

\section{Agradecimentos}

À Universidade do Estado da Bahia (UNEB), Fundação de Amparo à Pesquisa do Estado da Bahia (FAPESB), Embrapa Semiárido.

\section{Referências}

AGUIAR, R. W. S.; BRITO, D. R; OOTANI, M. A., FIDELIS, R. R.; PELUZIO, J. N. 2012. Efeito do dióxido do carbono, temperatura $\mathrm{e}$ armazenamento sobre sementes de soja e micoflora associada. Revista Ciência Agronômica, v. 43 , n. 3, p. 554-560.

AMBRIZZI, T.; ARAÚJO, M. 2012. Sumário Executivo do Volume 1 - Base Científica das Mudanças Climáticas. Contribuição do Grupo de Trabalho 1 para $01^{\circ}$ Relatório de Avaliação Nacional do Painel Brasileiro de Mudanças Climáticas. Rio de Janeiro: Painel Brasileiro de Mudanças Climáticas, 34p.

ANGELOTTI, F.; SÁ, I. B.; MELO, R. F. 2009. Mudanças climáticas e Desertificação no Semiárido Brasileiro. In: ANGELOTTI, F.; SÁ, I. B.; MENEZES, E. A.; PELLEGRINO G. Q.; Editores. Mudanças climáticas e desertificação no Semiárido brasileiro. Petrolina, PE: Embrapa Semiárido, Embrapa Informática Agropecuária.

ATKIN, O. K.; SCHEURWATER, I.; PONS, T. L. 2007. Respiration as a percentage of daily photosynthesis in whole plants is homeostatic at moderate, but not high, growth temperatures. New Phytologist, v. 174, n. 2, p. 367-380.

BROWN, J. H.; VALONE, T. J.; CURTIN, C. G. 1997. Reorganization of an arid ecosystem in response to recent climate change. Proceedings of the National Academy of Sciences, v. 94, n. 18, p. 9729-9733.

BUCKERIDGE, M. S. 2008. A biologia e as mudanças climáticas no Brasil. São Carlos: Ed. RiMa, 316p.

CARDOSO, V. J. M. 2011. Metodologia para análise da dependência térmica da germinação pelo modelo de graus-dia. Oecologia Australis, v. 15 , n. 2, p. 236-248.

CASTRO, P. R. C.; KLUGE, R. A.; PERES, L. E. P. 2005. Manual de fisiologia vegetal: teoria e prática. Piracicaba: Ed. Agronômica Ceres, 650p.

CENTRITTO, M.; LEE, H. S.; JARVIS, P. 1999. Increased growth in elevated $\left[\mathrm{CO}_{2}\right]$ : an early, short-term response? Global Change Biology, v. 5, n. 6, p. 623-633.

CRAFS-BRANDNER, S. J.; SALVUCCI, E. M.; 2000. Rubisco activase constrains the photosynthetic potential of leaves at high 
temperature and $\mathrm{CO}_{2}$. Proceedings of the National Academy of Sciences, v. 97, n. 24, p. 1343013435.

CUNHA, A. C. R.; OSUNA, JTA, QUEIROZ, S. R. O. D.; RIOS, A. P. S. 2009. Crescimento inicial de angico em função do substrato e luminosidade. Revista Magistra, v. 21, n. 3, p. 179-186.

FERREIRA, D. F. 2008. SISVAR: um programa para análises e ensino de estatística. Revista Symposium, v. 6, n. 2, p. 36-41.

HÄTTENSCHWILER, S.; MIGLIETTA, F.; RASCHI, A.; KÖRNER, C. 1997. Thirty years of in situ tree growth under elevated $\mathrm{CO}_{2}$ : a model for future forest responses? Global Change Biology, v. 3, n. 5, p. 463-471.

IPCC, 2014: Climate Change 2014: Synthesis Report. Contribution of Working Groups I, II and III to the Fifth Assessment Report of the Intergovernmental Panel on Climate Change. Geneva, 151p.

KOTOWSKI, F. 1926. Temperature relations to germination of vegetable seed. Proceedings of the American society for horticultural science, v. 23, p. 176-184, 1926.

LABOURIAU, L. G. 1983. A germinação das sementes. Monografia, Organização dos Estados Americanos. Programa Regional de Desenvolvimento Científico e Tecnológico. Série de Biologia, v. 24

LARCHER, W. 2006. Ecofisiologia Vegetal. São Paulo: Ed. Rima, 531p.

LORENZI, H. 2002. Árvores brasileiras: manual de identificação e cultivo de plantas arbóreas do Brasil. Nova Odessa: Ed. Instituto Plantarum, $368 \mathrm{p}$.

LORENZI, H.; MATOS, F. J. A. 2008. Plantas medicinais no Brasil: nativas e exóticas. Nova Odessa: Ed. Instituto Plantarum, 544 p.

MAGALHÃES, A. R. 2013. Drought proofing rural economies in semi-arid regions. Climate Adaptation Futures, pp. 294-300.

MAGALHÃES, A. R. 2009. As mudanças climáticas globais e a desertificação. In: ANGELOTTI, F.; SÁ, I. B.; MENEZES, E. A.; PELLEGRINO, G. Q. Editores. Mudanças climáticas e desertificação no Semiárido brasileiro. Petrolina, PE: Embrapa Semiárido, Embrapa Informática Agropecuária.

MAGUIRE, J. D. 1962. Speed of germination-aid in selection and evaluation for seedling emergence and vigor. Crop science, v. 2, n. 2, p. 176-177.

MAIA, G. N. 2006. Caatinga: árvores e arbustos e suas utilidades. São Paulo: D e Z computação.

MAIA, G.N. 2004. Caatinga: árvores e arbustos e suas utilidades. São Paulo: Ed. Leitura \& Arte, 413p.

MARENGO, J. A. 2006. Mudanças climáticas globais e seus efeitos sobre a biodiversidade: Caracterização do clima atual e definição das alterações climáticas para o território brasileiro ao longo do século XXI. Brasília: MMA.

MARTINEZ, C. A.; OLIVEIRA, E. A. D. de; MELLO, T. R. P.; ALZATE-MARIN, A. L. 2015. Respostas das plantas ao incremento atmosférico de dióxido de carbono e da temperatura. Revista Brasileira de Geografia Física, v. 8, p. 635-650.

MARTINS, C. C.; MACHADO, C. G.; NAKAGAWA, J. 2008. Temperatura e substrato para o teste de germinação de sementes de barbatimão (Stryphnodendron adstringens (Mart.) Coville (Leguminosae). Revista Árvore, v. 32, n. 4, p. 633- 639.

MEIADO, M. V. 2012. Germinação de cactos de ecossistemas nordestinos. Tese de Doutorado. Universidade Federal de Pernambuco. Recife, Pernambuco, Brasil. 142p.

OOI, M.K.J.; AULD, T. D.; DENHAM, A. J. 2009. Climate change and bet-hedging: interactions between increased soil temperatures and seed bank persistence. Global Change Biology, v. 15, p. 2375-2386.

PASSOS, M. A. A.; SILVA, F. J. B. C.; SILVA, E. C. A.; PESSOA, M. M. L.; SANTOS, R. C. 2008. Luz, substrato e temperatura na germinação de sementes de cedro-vermelho. Pesquisa Agropecuária Brasileira, v. 43, n. 2, p. 281-284.

PIO-CORRÊA, M. 1984. Dicionário de Plantas Úteis do Brasil e das Exóticas Cultivadas. Rio de Janeiro: Ministério da Agricultura, 777p.

REYENGA, P. J.; SMITH, D. S.; PASTURES, G. C. T. E. 1999. Impacts of global change on 
cropping in SW Queensland. Environmental Modelling and Software, v. 14, p. 297-306.

REZENDE, F. M. DE; SOUZA, A. P. DE; BUCKERIDGE, M. S.; FURLAN, C. M. 2015. Is guava phenolic metabolism influenced by elevated atmospheric $\mathrm{CO}_{2}$ ? Environmental Pollution, v. 196, p. 483-488.

SAGE, R. F. 2002. How terrestrial organism sense, signal, and response to carbon dioxide. Integrative and Comparative Biology, v. 42, n. 3, p. $469-480$.

SALAZAR, A.; GOLDSTEIN, G.; FRANCO, A. C.; MIRALLES-WILHELM, F. 2011. Timing of seed dispersal and dormancy, rather than persistent soil seed-banks, control seedling recruitment of woody plants in Neotropical savannas. Seed Science Research, v. 21, n. 2, p. 103-116.

SANTANA, D. G.; RANAL, M. A. 2000. Statistical analysis in germination. Revista Brasileira de Fisiologia Vegetal, v. 12, p. 205-237.

STRECK, N. A. 2005. Climate change and agroecosystems: the effect of elevated atmospheric $\mathrm{CO}_{2}$ and temperature on crop growth, development, and yield. Ciência Rural, v. 35, n. 3, p. $730-740$.

TAIZ, L.; ZEIGER, E. 2013. Fisiologia Vegetal. Porto Alegre: Artmed, 918p.
WALCK, J. L.; HIDAYATI, S. N.; DIXON, K. W.; THOMPSON, K.; POSCHLOD, P. 2011. Climate change and plant regeneration from seed. Global Change Biology, v. 17, n. 6, p. 2145-2161.

WALTHER, G. R.; POST, E.; CONVEY, P.; MENZEL, A.; PARMESAN, C.; BEEBEE, J. C. T.; FROMENTIN, J. M.; HOEGH-GULDBERG, O.; FRANZ BAIRLEIN, F. 2002. Ecological responses to recent climate change. Nature, v. 416 , n. 6879 , p. $389-395$.

WÜRTH, M. K. R.; WINTER, K.; KÖRNER, C. 1998. In situ responses to elevated $\mathrm{CO}_{2}$ in tropical forest understory plants. Functional Ecology, v. 12 , n. 6 , p. 886-895.

YAMASAKI, T.; YAMAKAWA, T.; YAMANE, Y.; KOIKE, H.; SATOH, K.; KATOH, S. 2002. Temperature acclimation of photosynthesis and related changes in photosystem II electron transport in winter wheat. Plant Physiology, v. 128, n. 3, p. 1087-1097.

ZAVALETA, E. S. 2006. Shrub establishment under experimental global changes in a California grassland. Plant Ecology, v. 184, n. 1, p. 53-63.

ZAVALETA, E.S.; SHAW, M. R.; CHIARIELLO, N. R.; THOMAS, B. D.; CLELAND, E. E.; FIELD, C. B.; MOONEY, H. A. 2003. Grassland responses to three years of elevated temperature, $\mathrm{CO}_{2}$, precipitation, and $\mathrm{N}$ deposition. Ecological Monographs, v. 73, n. 4, p. 585-604. 